\title{
Metabolic Score for Visceral Fat (METS-VF) Estimation - A Novel Cost-Effective Obesity Indicator for Visceral Adipose Tissue Estimation
}

This article was published in the following Dove Press journal: Diabetes, Metabolic Syndrome and Obesity: Targets and Therapy

\author{
Nitin Kapoor (1D ${ }^{1,2}$ \\ Stephen A Jiwanmall ${ }^{3}$ \\ Munaf B Nandyal ${ }^{3}$ \\ Dheeraj Kattula ${ }^{3}$ \\ Sandhiya Paravathareddy ${ }^{\prime}$ \\ Thomas V Paul' \\ John Furler ${ }^{4}$ \\ Brian Oldenburg ${ }^{2}$ \\ Nihal Thomas' \\ 'Department of Endocrinology, Diabetes \\ and Metabolism Christian Medical \\ College \& Hospital, Vellore, Tamil Nadu, \\ India; ${ }^{2}$ Non-Communicable Disease Unit, \\ Melbourne School of Population and \\ Global Health, University of Melbourne, \\ Melbourne, Australia; ${ }^{3}$ Department of \\ Psychiatry, Christian Medical College \& \\ Hospital, Vellore, Tamil Nadu, India; \\ ${ }^{4}$ Department of General Practice, \\ University of Melbourne, Melbourne, \\ Australia
}

Background: Visceral adipose tissue (VAT) assessment is limited in clinical practice due to expensive, time consuming and limited availability of MRI and DXA machines. We explored the utility of a recently developed Metabolic Score for Visceral Fat (METS-VF) to assess VAT in south Asian individuals with morbid obesity.

Patients and Methods: Individuals with BMI $\geq 35 \mathrm{~kg} / \mathrm{m}^{2}$ aged between 30 and 60 years were randomly selected from a database of individuals with morbid obesity, attending a multi-disciplinary bariatric clinic in a tertiary care teaching hospital in southern India. Body composition was assessed by using a Hologic Discovery A dual-energy X-ray absorptiometry (DXA) machine. METS-VF was used to estimate VAT by using a previously published algorithm.

Results: The mean age and body mass index of the study subjects $(\mathrm{N}=350)$ were 38.2 years and $40.1 \mathrm{~kg} / \mathrm{m}^{2}$. The MET-VF score performed satisfactorily (AUC of 0.78 (95\% CI $0.72-$ $0.85)$ ) for predicting an increased visceral adipose tissue (VAT area $\geq 163 \mathrm{~cm}^{2}$ ) as detected by DXA. A METS-VF value of 7.3 was found to have a good sensitivity and reasonable specificity in predicting elevated VAT in this population.

Conclusion: This is the first study to validate the utility of METS-VF as a surrogate measure of visceral adiposity in south Indian individuals with morbid obesity. Given the simplicity, easy availability, reliability and inexpensive nature of this obesity indicator, it may find its widespread use in lower middle-income countries.

Keywords: metabolic obesity, morbid obesity, visceral adipose tissue

\section{Introduction}

The global prevalence of obesity has increased rapidly over the last two decades and has now reached pandemic proportions. A recent study by the Global Burden Of Disease - Obesity Collaborators, has described the problem of obesity in relation to high body-mass index (BMI), in 195 countries between 1990 and 2015. ${ }^{1}$ Their findings reveal that an elevated BMI accounted for about 4.0 million deaths worldwide in 2015 and more than two thirds of these deaths were due to cardiovascular disease. ${ }^{1}$ Moreover, it has also been noted that the prevalence of individuals with morbid obesity is increasing more markedly than individuals in other weight groups. ${ }^{2}$ Lower middle income countries (LMIC's) like India show similar trends and in some states up to onethird of the general population have either overweight or obesity. ${ }^{1,3}$

However, not all individuals with obesity develop a similar course of metabolic complications. ${ }^{4}$ This is also true for individuals with morbid obesity and may partly
Correspondence: Nitin Kapoor

Email nitin.endocrine@gmail.com
Diabetes, Metabolic Syndrome and Obesity: Targets and Therapy 2020:13 326|-3267 
be due to the inability of BMI to identify individuals with high metabolic risk. ${ }^{5}$ The measures of central obesity like waist circumference, waist-hip ratio and waist height ratio may be relatively better but unable to precisely estimate visceral adipose tissue. ${ }^{6}$ Moreover, it can be difficult to accurately measure waist circumference in individuals with morbid obesity. ${ }^{7}$ Moreover, these obesity indicators are unable to differentiate between the presence of the socalled "good subcutaneous fat" versus metabolically toxic visceral or ectopic fat, at the site of measurement. ${ }^{8}$ The gold standard to assess visceral adipose tissue (VAT) is an Magnetic Resonance Imaging (MRI) scan which is not only expensive, has limited availability and has size limitations but it also requires considerable expertise and time to interpret. The next best option of VAT estimation is a Dual energy X-RAY absorptiometry (DXA) Scan, which has a much lower radiation exposure but it is again limited by its availability, cost and lack of ethnicity specific normative data for comparison. ${ }^{5}$ This calls for development of novel tools to accurately estimate visceral adipose tissue which are cost effective, easily available, simple, safe, reliable and ethnically validated. Some tools developed in the past including VAT=TAAT-SAAT model and apVAT - anthropometrically predicted visceral adipose tissue, have attempted to estimate VAT but have not been extensively validated in the south Asian population. ${ }^{9-11}$

To address this research gap we explored the utility of a recently developed Metabolic Score for Visceral Fat (METS-VF) which uses easily available clinical parameters against VAT as assessed by a DXA scan in south Asian individuals with morbid obesity. ${ }^{12}$ The complexities of assessing VAT despite its importance in predicting metabolic complications are akin to measurement of GFR (glomerular filtration rate) in patients with chronic kidney disease, which in actual is a laborious process but can be estimated by validated algorithms to measure eGFR as is commonly used in clinical practice.

\section{Patients and Methods}

This was a cross-sectional study conducted over a period of one year. Study subjects were recruited from a database of individuals with morbid obesity attending a multidisciplinary bariatric clinic in a tertiary care teaching hospital in Southern India. Individuals with morbid obesity $\left(B M I \geq 35 \mathrm{~kg} / \mathrm{m}^{2}\right.$ ) aged between 30 and 60 years were selected by simple random sampling from the database. Patients with endocrinopathies, syndromic causes of obesity, on medications that increase weight (example glucocorticoids) and who declined consent were not included in the study. This study was approved by the Institutional Review Board - Independent Ethics committee of Christian Medical College, Vellore, India, vide IRB Min No 10,146 dated 22.06.2016. All participants provided informed consent, and that this study complied with the Declaration of Helsinki.

Demographic details and a detailed history to assess comorbidities associated with obesity were assessed using a pre-structured detailed questionnaire. Anthropometric measurements including height (in centimeters), weight (in Kilograms), waist circumference (in centimeters), hip circumference (in centimeters), waist hip ratio (WHR) and waist height ratio (WHtR) were obtained using predefined standardized techniques. Body composition including body fat percentage, Fat height index (Fat/height ${ }^{2}$ ) and VAT (visceral fat area in $\mathrm{cm}^{2}$ ) was estimated using a whole-body dualenergy X-ray absorptiometry (DXA) scan using a Hologic DXA QDR 4500 Discovery A machine with a coefficient of variation $(\mathrm{CV})$ of $2 \%$. All participants also underwent the following biochemical tests: fasting plasma glucose, 2 hour post prandial plasma glucose (enzymatic method), hemoglobin A1c (high-performance liquid chromatography) and fasting lipid profile (enzymatic colorimetric assay-oxidase peroxidase method). METS-VF was used to estimate VAT by combining the non-insulin-based METS-IR index, waistheight ratio (WHtr), age and sex by using the previously published algorithm. METS-IR was calculated using fasting serum triglyceride, high density lipoprotein cholesterol and fasting glucose. ${ }^{12}$

Diabetes was defined according to the criteria proposed by the American Diabetes Association, which states that individuals with a fasting plasma glucose value $\geq 126 \mathrm{mg} / \mathrm{dl}$ and/or 2-h plasma glucose value of $\geq 200 \mathrm{mg} / \mathrm{dl}$ and/or HbAlc $>6.5 \%$ have diabetes. Patients taking anti-diabetic medications were also considered as having diabetes. Patients with a fasting plasma glucose value between $100 \mathrm{mg} / \mathrm{dl}$ and $125 \mathrm{mg} / \mathrm{dl}$ with a 2 hour plasma glucose $<140 \mathrm{mg} / \mathrm{dl}$ (impaired fasting glucose) and/or a 2 hour plasma glucose between $140 \mathrm{mg} / \mathrm{dl}$ and $200 \mathrm{mg} / \mathrm{dl}$ with a fasting plasma glucose less than $100 \mathrm{mg} /$ dl (impaired glucose tolerance) were diagnosed to have pre-diabetes. ${ }^{13}$ Hypertension was defined in individuals with a systolic BP of $\geq 140 \mathrm{mmHg}$ and/or diastolic BP $\geq$ $90 \mathrm{mmHg}$ and/or currently taking BP lowering medications. Patients with pre-hypertension were defined if they had a systolic BP between 120 and $139 \mathrm{mmHg}$ and/or 
diastolic BP between 80 and $89 \mathrm{mmHg}$ and were not taking blood pressure lowering medications. ${ }^{14}$ Dyslipidemia was defined as in individuals who were taking lipid-lowering medications and/or had a high total cholesterol $(>200 \mathrm{mg} / \mathrm{dl})$ and/or high LDL cholesterol $(>100 \mathrm{mg} / \mathrm{dl})$ and/or low HDL cholesterol $(<40 \mathrm{mg} / \mathrm{dl}$ in men and $<50 \mathrm{mg} / \mathrm{dl}$ in women) and/or high triglycerides $(>200 \mathrm{mg} / \mathrm{dl})^{15}$

Based on the presence of co-morbidities an Edmonton Obesity Staging System score was also calculated for each of the participants. The EOSS system consists of 5 scores based on the physical, psychological and functional disability of each patient. ${ }^{4,16}$ The EOSS level is categorized reckoning the highest-stage risk factor present in each individual. Stage 0: no obesity-related risk factors; Stage 1: mild risk factors (example in the metabolic category having prediabetes, prehypertension); Stage 2: Moderate symptoms often needing medical treatment (example in the metabolic category having diabetes, hypertension); Stage 3: functional symptoms and organ damage affecting the living standard (example in the metabolic category having early stages of diabetic nephropathy); Stage 4: severe disabilities from obesity (example in the metabolic category having end stage nephropathy).

Data analysis was done using SPSS 17.0 version. Descriptive statistics including continuous demographic variables and anthropometric indicators were reported using mean \pm standard deviation (SD). Categorical demographic variables, presence of comorbidities and EOSS staging were reported using frequency and percentage. The correlation between 2 continuous variables (METS-VF and VAT area) was performed using Pearson correlation coefficient. The METS-VF was plotted as a continuous variable against increased visceral adipose tissue estimated by DXA. A VAT area $\geq 163 \mathrm{~cm}^{2}$ was considered as increased, based on a previously published study. ${ }^{17}$ The sensitivity, the specificity, and the area under the curve for METS-VF predicting the presence or absence of increased visceral adipose tissue were calculated. Receiver operating characteristics (ROC) curves were constructed using different cut-offs of METSVF, to derive the cut-offs which could best predict increased VAT area estimated by DXA $\left(\geq 163 \mathrm{~cm}^{2}\right)$.

\section{Results}

A total of 350 individuals were recruited for this study. Their mean ( \pm SD) age was 38.2 ( \pm 13.2) years. Seventy percent of the recruited participants were women (Women, $\mathrm{N}=249$. Men, $\mathrm{N}=101$ ). The mean ( $\pm \mathrm{SD}$ ) body mass index of the study subjects was $40.1( \pm 6.2) \mathrm{kg} / \mathrm{m}^{2}$.
191/350 (55\%) had hyperglycemia (Type 2 Diabetes Mellitus (122/350) and Pre- Diabetes (69/350)), 233/350 (67\%) had elevated blood pressure (hypertension91/350) and pre-hypertension (142/350), 221/350 (63.1\%) had dyslipidemia, 148/350 (42.2\%) had osteoarthritis and 253/350 $(72 \%)$ had suspected OSA. $68 \%$ of individuals had an EOSS stage 2 and 3. 37.5\% (131/350) of the subjects had a high visceral adipose tissue area based on the definition defined before. Their demographic details, anthropometric measurements and comorbidities between subjects with a high and low VAT are summarized in Table 1.

The distribution of their EOSS staging is shown in Figure 1. There was a strong correlation between METSVF and the VAT area as measured by DXA $(r=+0.517$ [95\% CI: 0.31-0.67]; p value <0.001). Since METS-VF predicts visceral fat as a logarithm of fat mass. We transformed METS-VF values to fat grams, which provided similar values $(\mathrm{r}=+0.533$ [95\% CI: $0.38-0.72$ ]; $\mathrm{p}$ value $<0.001)$ A graph showing the correlation between visceral fat area and METS-VF is shown in Figure 2.

The METS-VF along with other anthropometric parameters was plotted as a continuous variable against increased visceral adipose tissue $\left(>163 \mathrm{~cm}^{2}\right)$ estimated by DXA for a ROC analysis. METS-VF had the best diagnostic accuracy of predicting VAT scores as compared to other anthropometric parameters (Figure 3). The area under curve for the ability of METS-VF to predict VAT was 0.782 (95\% CI: $0.72-0.85$; p value $<0.001)$. A METSVF value of 7.3 was found to have a sensitivity of $86.2 \%$ (95\% CI: 80.69-90.63\%) and a specificity of 59.8\% (95\% CI $-51.47-67.85 \%$ ) to predict increased VAT. The positive predictive value was $74.79 \%$ (95\% CI:70.73-78.45\%) and the negative predictive value was found to be $75.86 \%(95 \%$ CI: $68.5-81.96 \%$ )

\section{Discussion}

In this study, 350 individuals with morbid obesity were recruited from a bariatric clinic database in a tertiary care center in southern India. The mean age and body mass index of the study subjects was 38.2 years and $40.1 \mathrm{~kg} / \mathrm{m}^{2}$. Majority of the patients $(68 \%)$ were categorized to have EOSS stage 2 and 3. The MET-VF score performed satisfactorily (AUC of 0.782 (95\% CI 0.72-0.85)) for predicting an increased visceral adipose tissue (VAT area $\geq$ $163 \mathrm{~cm}^{2}$ ) as detected by DXA. A METS-VF value of 7.3 was found to have a good sensitivity and reasonable specificity in predicting elevated VAT in this south Indian population. 
Table I Baseline Characteristics of the Study Subjects Between Subjects with High vs Low Visceral Adipose Tissue Area

\begin{tabular}{|c|c|c|c|}
\hline $\begin{array}{l}\text { Parameter Studied } \\
N=350\end{array}$ & $\begin{array}{l}\text { Individuals with High VAT } \\
N=|3|\end{array}$ & $\begin{array}{l}\text { Individuals with Low VAT } \\
\mathrm{N}=219\end{array}$ & $\mathbf{P}$ value \\
\hline Age in years, Mean(SD) & $39.2 \pm 11.6$ & $36.7 \pm 13.2$ & 0.07 \\
\hline Gender(M:F) & $38: 93(\sim 1: 2)$ & $63: 156(\sim 1: 2)$ & 0.95 \\
\hline Height in cm, Mean(SD) & $160.1(9.2)$ & $161.6(9.5)$ & 0.14 \\
\hline Weight in kg, Mean(SD) & $106.6(17.9)$ & $99.5(18.3)$ & 0.005 \\
\hline Body Mass Index in $\mathrm{kg} / \mathrm{m}^{2}$, Mean(SD) & $4 I .5(5.7)$ & $38.1(6.2)$ & 0.001 \\
\hline Waist circumference in $\mathrm{cm}$, Mean(SD) & $126.0(12.7)$ & $119.1(13.9)$ & 0.001 \\
\hline Waist height ratio, Mean(SD) & $0.75(0.06)$ & $0.69(0.08)$ & $<0.001$ \\
\hline Hip circumference in cm, Mean(SD) & $122.4(8.6)$ & $120.1(\mid 1.9)$ & 0.06 \\
\hline Waist hip ratio, Mean(SD) & $0.98(0.09)$ & $0.97(0.08)$ & 0.28 \\
\hline Type 2 diabetes, $\mathrm{n}(\%)$ & $59(45.1 \%)$ & $63(28.7 \%)$ & $<0.001$ \\
\hline Hypertension, n(\%) & $47(35.8 \%)$ & $44(21.0 \%)$ & 0.002 \\
\hline Dyslipidemia, n(\%) & $73(55.7 \%)$ & $75(34.2 \%)$ & $<0.001$ \\
\hline
\end{tabular}

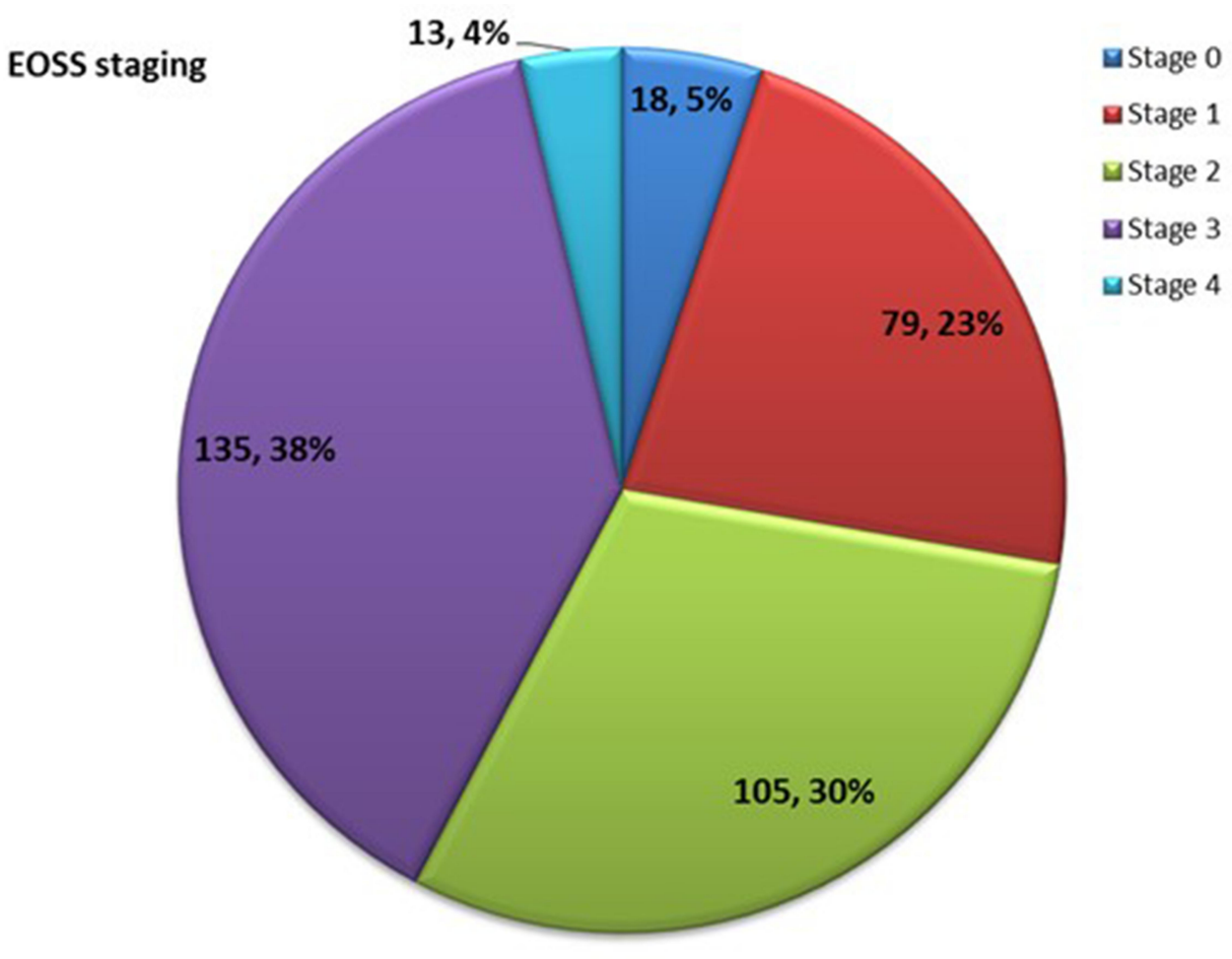

Figure I EOSS Staging (Edmonton obesity staging system) of the study subjects.

In comparison to data from other bariatric centers across the world, our clinic represents a relatively young population (mean age of 38.2 years). The mean age of patients in an Irish bariatric clinic cohort was 54 years and in an obesity clinic population from New Zealand was 46 years. Similar to our study, the mean age in a bariatric center from Saudi Arabia 


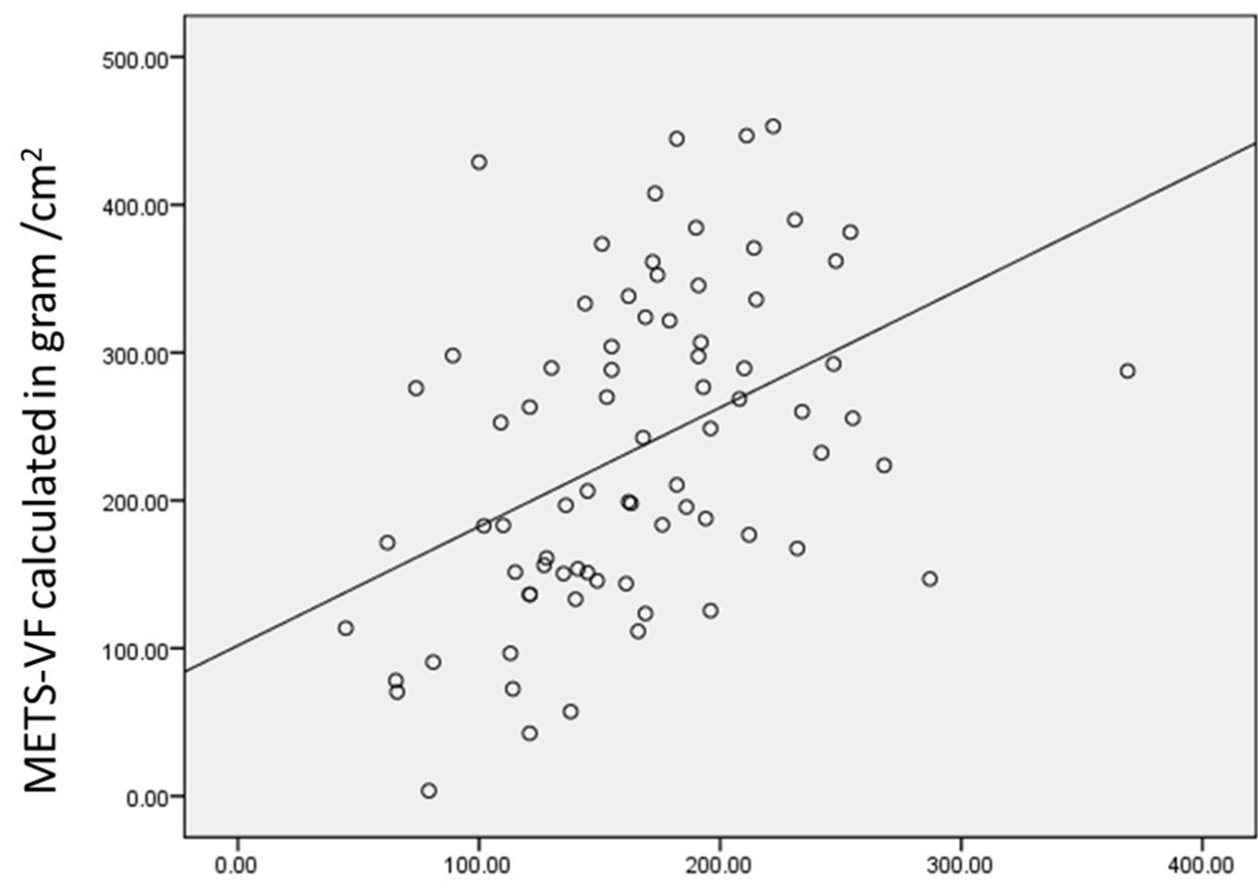

VAT Area as estimated by DXA scan in gram $/ \mathrm{cm}^{2}$

Figure 2 Correlation between METS-VF and VAT area.

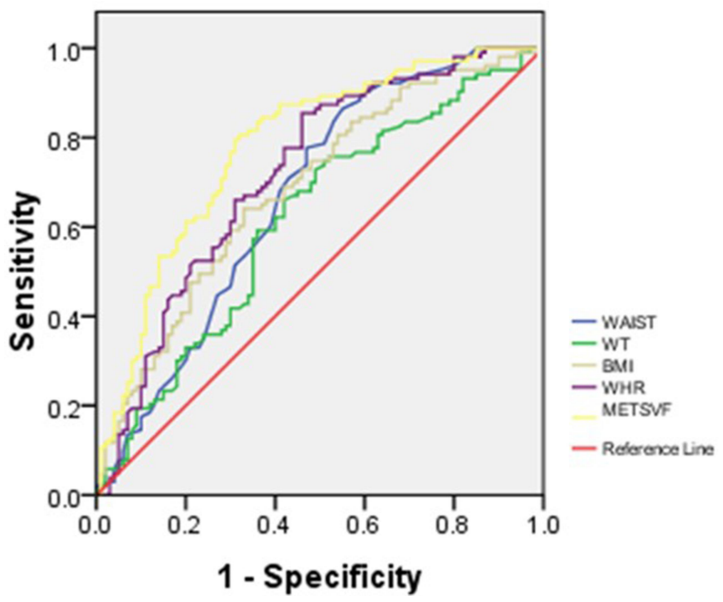

\begin{tabular}{|l|l|l|l|}
\hline Anthropometric measure & $\begin{array}{l}\text { Area under } \\
\text { the curve }\end{array}$ & $\begin{array}{l}\text { 95\% confidence } \\
\text { interval limits }\end{array}$ & P value \\
\hline METS-VF & 0.782 & $0.718-0.846$ & $<0.001$ \\
\hline Waist Height ratio(WHR) & 0.721 & $0.651-0.792$ & $<0.001$ \\
\hline Body mass Index(BMI) & 0.686 & $0.613-0.759$ & $<0.001$ \\
\hline Waist circumference (WAIST) & 0.669 & $0.594-0.744$ & $<0.001$ \\
\hline Weight (WT) & 0.617 & $0.539-0.694$ & 0.004 \\
\hline
\end{tabular}

Figure 3 ROC analysis to test the accuracy of different clinical indicators to predict visceral adiposity in individuals with morbid obesity.

was 37.2 years. ${ }^{18-20}$ This age difference could be explained by the mean population age and the increased life expectancy in these countries. However, the mean body mass index at our center $\left(40.1 \mathrm{~kg} / \mathrm{m}^{2}\right)$ was also relatively lower compared to other centers $\left(47.1 \mathrm{~kg} / \mathrm{m}^{2}\right.$ (Irish) and $47.4 \mathrm{~kg} / \mathrm{m}^{2}$ (New Zealand)) but similar to a center in Saudi Arabia (39.6 kg/ $\mathrm{m}^{2}$ ). The other socio-demographic indicators are comparable to other clinic database. ${ }^{18-20}$
Despite a younger age and lower body mass index, the proportion of individuals with metabolic complications in our population (type 2 diabetes mellitus - 34.8\%; hypertension $-26 \%$; dyslipidemia $-42 \%$ ) were much higher than what is reported in bariatric centers representing a European population (type 2 diabetes $-25 \%$, hypertension $22.4 \%$ and dyslipidemia $-21 \%) .{ }^{18}$ The EOSS staging in our study subjects was comparable to other study 
populations in similar settings (Figure 1). Two-third of our study subjects were in stage 2 and stage 3(68\%), which is comparable to other obesity cohorts as in Germany $(70 \%$ in stage 2), Brazil (86.5\% in stage 2), Canada (84\% in stage 3$)^{21-23}$

These ethnicity specific differences, are probably secondary to the previously reported higher propensity of the South Asian population to develop metabolic complications at a lower degree of obesity. ${ }^{5-8}$ Several hypotheses that may explain this include a lower birth weight, lower capacity of subcutaneous fat resulting in fat overflow to ectopic sites, higher visceral adiposity and underlying genetic predisposition in the South Asian individuals. ${ }^{24-}$ 28 This further emphasizes the need to assess visceral adipose tissue in a clinical context before suggesting life changing management decisions in patients with morbid obesity.

Though visceral adipose tissue assessment is possible by different methods including magnetic resonance imaging and computed tomography scans, these have their own limitations as listed before. In a quest to have an easy to access, simple, accurate measure of VAT that can be derived using common clinically available parameters, METS-VF was recently developed using a mathematical model. ${ }^{12}$ This model used non-linear fits of individual crude and log-transformed variables aiming to maximize the explained variance of its individual components. The main predictors of VAT including insulin resistance, anthropometric measure of body-fat distribution -waist height ratio (WHtr), age, and sex were used. $^{29,30}$ Furthermore, since it uses simple and commonly available clinical parameters, it can be of much use in cost restrained settings. There are other tools that have been developed to estimate VAT. These include the VAT=TAATSAAT model and apVAT - anthropometrically predicted visceral adipose tissue. ${ }^{10,11}$ Though these have shown good sensitivity and specificity in selected populations, there is paucity of literature regarding validation in south Asian populations. ${ }^{9}$

In the present study, we found that METS-VF correlates well with VAT values as estimated by DXA. Moreover, it has a good area under the curve and can reliably predict VAT area in individuals with morbid obesity with a good sensitivity and an optimal specificity. This is the first study to show its utility in Indian patients with morbid obesity, who have a unique phenotype and are prone to metabolic complications at a lower grade of obesity. The main limitations of this study include its cross-sectional design and a hospital-based study population, which limits its generalizability to the general population. In addition, the components of METS-VF including waist-height ratio and METS-IR are indirect measures of the morphologic phenotype and insulin resistance. We also acknowledge that further longitudinal studies are needed to study the utility of this surrogate to predict response to various therapeutic options.

To conclude, this study suggests that the unique South Asian phenotype of relatively high body fat percentage is not only seen in apparently lean individuals but also in people with morbid obesity. ${ }^{31,32}$ Individuals with morbid obesity in this study are younger, have a relatively lower BMI and more metabolic complications when compared to similar bariatric centers in other ethnicities. This is the first study to validate the utility of METS-VF as a surrogate measure of truncal fat and estimated visceral adiposity in south Indian patients with morbid obesity. Given the simplicity, easy availability, and reliability of this obesity indicator, it may find widespread use in lower middleincome countries.

\section{Disclosure}

The authors report no conflicts of interest for this work.

\section{References}

1. Afshin A, Forouzanfar MH, Reitsma MB, et al. Health effects of overweight and obesity in 195 countries over 25 years. $N$ Engl J Med. 2017;377(1):13-27.

2. Sturm R, Hattori A. Morbid obesity rates continue to rise rapidly in the United States. Int J Obes (Lond). 2013;37(6):889-891. doi:10.1038/ ijo.2012.159

3. Kalra S, Unnikrishnan A. Obesity in India: the weight of the nation. $J$ Med Nutr Nutracenticals. 2012;1(1):37. doi:10.4103/2278019X.94634

4. Padwal RS, Pajewski NM, Allison DB, Sharma AM. Using the Edmonton obesity staging system to predict mortality in a population-representative cohort of people with overweight and obesity. CMAJ. 2011;183(14):E1059-66. doi:10.1503/cmaj.110387

5. Kapoor N, Furler J, Paul TV, Thomas N, Oldenburg B. The BMI-adiposity conundrum in South Asian populations: need for further research. J Biosoc Sci. 2019;51(4):619-621. doi:10.1017/ S0021932019000166

6. Kapoor N, Lotfaliany M, Sathish T, et al. Obesity indicators that best predict type 2 diabetes in an Indian population: insights from the Kerala diabetes prevention program. J Nutr Sci. 2020;9. doi:10.1017/ jns.2020.8

7. Kapoor N, Furler J, Paul TV, Thomas N, Oldenburg B. Ethnicityspecific cut-offs that predict co-morbidities: the way forward for optimal utility of obesity indicators. J Biosoc Sci. 2019;51 (4):624-626. doi:10.1017/S0021932019000178

8. Kapoor N, Furler J, Paul TV, Thomas N, Oldenburg B. Normal weight obesity: an underrecognized problem in individuals of South Asian Descent. Clin Ther. 2019;41(8):1638-1642. doi:10.1016/j. clinthera.2019.05.016 
9. Brown JC, Harhay MO, Harhay MN. Anthropometrically-predicted visceral adipose tissue and mortality among men and women in the third national health and nutrition examination survey (NHANES III). Am J Human Biol. 2017;29(1):e22898. doi:10.1002/ajhb.22898

10. Brown JC, Harhay MO, Harhay MN. Anthropometrically predicted visceral adipose tissue and blood-based biomarkers: a cross-sectional analysis. Eur J Nutr. 2018;57(1):191-198. doi:10.1007/s00394-0161308-8

11. Samouda H, Dutour A, Chaumoitre K, Panuel M, Dutour O, Dadoun F. VAT=TAAT-SAAT: innovative anthropometric model to predict visceral adipose tissue without resort to CT-Scan or DXA. Obesity (Silver Spring, Md). 2013;21(1):E41-50. doi:10.1002/ oby. 20033

12. Bello-Chavolla OY, Antonio-Villa NE, Vargas-Vazquez A, et al. Metabolic Score for Visceral Fat (METS-VF), a novel estimator of intra-abdominal fat content and cardio-metabolic health. Clin Nutr (Edinburgh, Scotland). 2019;39(5):1613-1621.

13. American Diabetes Association. 2. Classification and diagnosis of diabetes: standards of medical care in diabetes-2020. Diabetes Care. 2020;43(Suppl 1):S14-s31. doi:10.2337/dc20-S002

14. James PA, Oparil S, Carter BL, et al. 2014 evidence-based guideline for the management of high blood pressure in adults: report from the panel members appointed to the Eighth Joint National Committee (JNC 8). JAMA. 2014;311(5):507-520. doi:10.1001/jama.2013.284427

15. National Cholesterol Education Program (NCEP). Expert Panel on Detection, Evaluation, and Treatment of High Blood Cholesterol in Adults (Adult Treatment Panel III). Third report of the National Cholesterol Education Program (NCEP) expert panel on detection, evaluation, and treatment of high blood cholesterol in adults (adult treatment panel III) final report. Circulation. 2002;106(25):3143. doi:10.1161/circ.106.25.3143

16. Martinez Urbistondo D, Martinez JA. Usefulness of the Edmonton obesity staging system to develop precise medical nutrition. Rev Clin Esp. 2017;217(2):97-98. doi:10.1016/j.rce.2017.01.001

17. Nicklas BJ, Penninx BW, Ryan AS, Berman DM, Lynch NA, Dennis KE. Visceral adipose tissue cutoffs associated with metabolic risk factors for coronary heart disease in women. Diabetes Care. 2003;26(5):1413-1420. doi:10.2337/diacare.26.5.1413

18. Fang CEH, Crowe C, Murphy A, O’Donnell M, Finucane FM. Crosssectional study of the association between skin tags and vascular risk factors in a bariatric clinic-based cohort of Irish adults with morbid obesity. BMC Res Notes. 2020;13(1):156. doi:10.1186/s13104-02005006-4

19. Alghamdi RQ. A randomized controlled trial of a 12-week intensive lifestyle intervention program at a primary care obesity clinic for adults in western Saudi Arabia. Saudi Med J. 2017;38(8):837-845. doi:10.15537/smj.2017.8.20553

20. Shilton H, Gao Y, Nerlekar N, Evennett N, Ram R, Beban G. Preoperative bariatric clinic attendance is a predictor of post-operative clinic attendance and weight loss outcomes. Obes Surg. 2019;29 (7):2270-2275. doi:10.1007/s11695-019-03843-2
21. Ogassavara NC, Magalhaes Dias JG, Pajecki D, de Oliveira Siqueira J, Santo MA, Tess BH. The Edmonton obesity staging system: assessing a potential tool to improve the management of obesity surgery in the Brazilian public health services. Surg Obes Relat Dis. 2020;16(1):40-47. doi:10.1016/j.soard.2019.10.021

22. Chiappetta S, Stier C, Squillante S, Theodoridou S, Weiner RA. The importance of the Edmonton obesity staging system in predicting postoperative outcome and 30-day mortality after metabolic surgery. Surg Obes Relat Dis. 2016;12(10):1847-1855. doi:10.1016/j. soard.2016.02.042

23. Canning KL, Brown RE, Wharton S, Sharma AM, Kuk JL. Edmonton obesity staging system prevalence and association with weight loss in a publicly funded referral-based obesity clinic. J Obes. 2015;2015:619734. doi:10.1155/2015/619734

24. Anand SS, Tarnopolsky MA, Rashid S, et al. Adipocyte hypertrophy, fatty liver and metabolic risk factors in South Asians: the Molecular Study of Health and Risk in Ethnic Groups (mol-SHARE). PLoS One. 2011;6(7):e22112-e. doi:10.1371/journal.pone.0022112

25. Thomas N, Grunnet LG, Poulsen P, et al. Born with low birth weight in rural Southern India: what are the metabolic consequences 20 years later? Eur J Endocrinol. 2012;166(4):647-655. doi:10.1530/ EJE-11-0870

26. Thomas N, Bygbjerg IB. Does being born low birth weight affect the ability to exercise? Indian J Endocrinol Metab. 2016;20(6):741-743. doi:10.4103/2230-8210.192920

27. Kapoor N, Chapla A, Furler J, et al. Genetics of obesity in consanguineous populations-A road map to provide novel insights in the molecular basis and management of obesity. EBioMedicine. 2019;40:33-34. doi:10.1016/j.ebiom.2019.01.004

28. Chan Z, Ding C, Chooi YC, et al. Ectopic fat and aerobic fitness are key determinants of glucose homeostasis in nonobese Asians. Eur $J$ Clin Investig. 2019;49(5):e13079.

29. Amato MC, Giordano C, Galia M, et al. Visceral Adiposity Index: a reliable indicator of visceral fat function associated with cardiometabolic risk. Diabetes Care. 2010;33(4):920-922. doi:10.2337/dc091825

30. Brundavani V, Murthy SR, Kurpad AV. Estimation of deep-abdominal-adipose-tissue (DAAT) accumulation from simple anthropometric measurements in Indian men and women. Eur J Clin Nutr. 2006;60(5):658-666. doi:10.1038/sj.ejen.1602366

31. Kapoor N, Lotfaliany M, Sathish T, et al. Effect of a peer-led lifestyle intervention on individuals with normal weight obesity: insights from the Kerala diabetes prevention program. Clin Ther. 2020. doi:10.1016/j.clinthera.2020.06.007

32. Kapoor N, Lotfaliany M, Sathish T, et al. Prevalence of normal weight obesity and its associated cardio-metabolic risk factors Results from the baseline data of the Kerala Diabetes Prevention Program (KDPP). PLoS One. 2020;15(8):e0237974. doi:10.1371/ journal.pone.0237974

Diabetes, Metabolic Syndrome and Obesity: Targets and Therapy

Dovepress

\section{Publish your work in this journal}

Diabetes, Metabolic Syndrome and Obesity: Targets and Therapy is an international, peer-reviewed open-access journal committed to the rapid publication of the latest laboratory and clinical findings in the fields of diabetes, metabolic syndrome and obesity research. Original research, review, case reports, hypothesis formation, expert opinion and commentaries are all considered for publication. The manuscript management system is completely online and includes a very quick and fair peer-review system, which is all easy to use. Visit http://www.dovepress.com/testimonials.php to read real quotes from published authors. 